\title{
Estudio de la Combustión Espontánea del Carbón durante el Acopio en la Mina El Hatillo, ubicada en Cesar, Colombia
}

\author{
Gail A. Gutiérrez ${ }^{(1)}$, Daniel A. Cotes ${ }^{(1) *}$ y Marlon J. Bastidas ${ }^{(1,2)}$ \\ (1) Grupo de Investigación en Desarrollo de Estudios y Tecnologías Ambientales del Carbono DESTACAR, \\ Universidad de La Guajira, Riohacha-Colombia. (e-mail: galbeirog@uniguajira.edu.co; \\ dcotesg@uniguajira.edu.co; marlonjoseb@uniguajira.edu.co) \\ (2) Programa de Ingeniería Agroindustrial, Universidad Popular del Cesar, Bloque A, Oficina 205, \\ Sede Hurtado, Valledupar-Colombia.
}

* Autor a quien debe ser dirigida la correspondencia

Recibido Mar. 14, 2018; Aceptado May. 25, 2018; Versión final Jul. 10, 2018, Publicado Dic. 2018

\begin{abstract}
Resumen
Este documento revela la fenomenología de los carbones acopiados expuestos a factores atmosféricos mediante ensayos experimentales. Se presenta una simulación del modelo matemático de Frank Kamenestkii para determinar la altura óptima de las pilas y el tiempo de ignición en base a las propiedades del carbón. La caracterización de las muestras de carbón mostró que, entre los indicadores de calidad del carbón, el poder calorífico oscila entre 6038 y $6850 \mathrm{cal} / \mathrm{g}$, las cenizas entre 1.8 y $10.4 \%$ y la humedad entre 8.8 y $11.2 \%$. Estos números indican que se trata de un carbón de buena calidad térmica, propenso a alcanzar los límites críticos para la combustión espontánea. El estudio concluye que la fenomenología de la combustión espontánea se le atribuye a una reacción química entre el carbón y el oxígeno, generando energía en forma de calor, que es transportada por los mecanismos de transferencia de energía en una matriz porosa de carbón.
\end{abstract}

Palabras clave: carbón; centros de acopios; combustión espontánea.

\section{Research of the Coal Spontaneous Combustion on Stockpiles in The Hatillo Mine, located in Cesar in Colombia}

\begin{abstract}
This paper reveals the phenomenon that occurs in the stockpiles exposed to atmosphere factors by experimental assay. It presents a simulation of Frank Kamenestkii mathematical model for determining the stockpiles ideal height and the ignition time based on the coal properties. This research can be used as a parameter for improving the methodology in the stockpiles. The coal samples characterization showed that among the coal quality indicators, the calorific value ranges from 6038 to $6850 \mathrm{cal} / \mathrm{g}$, ash between 1.8 and $10.4 \%$ and humidity between 8.8 and $11.2 \%$, indicating a coal of good thermal quality, prone to reaching critical limits for spontaneous combustion. The study concludes that the spontaneous combustion phenomenology is attributed to a chemical reaction between coal and oxygen, generating energy in the form of heat, which is transported by energy transference mechanisms in a coal porous matrix.
\end{abstract}

Keywords: coal; stockpiles; spontaneous combustion. 


\section{INTRODUCCIÓN}

La combustión espontánea del carbón es un importante problema para la minería, debido a que representa pérdidas económicas, dificultades en la seguridad de las operaciones y consecuencias ambientales (Arisoy y Akgun, 1994; Arisoy, et al., 2006). En este documento se hace énfasis en la presencia del fenómeno durante el almacenamiento de carbón que se caracteriza por acumular grandes volúmenes durante largos periodos de tiempo. Sin embargo, este fenómeno puede ocurrir en minería subterránea, frentes de explotación y transporte a largas distancias (Carras y Young, 1994), además, es una preocupación mundial por la producción de gases invernadero (Pone, et al., 2007). Una de las etapas en la minería del carbón es la exposición de este combustible fósil a la atmósfera mediante centros de almacenamiento (Pone, et al., 2007), Arregocés et al (2016) determinaron que el almacenamiento de carbón industrial contribuye de forma significativa en las contribuciones de PM10 a las concentraciones ambientales diarias, debido a las condiciones meteorológicas, formas de las pilas y complejidad del terreno. También, se destaca que la mayoría de los tipos de carbones pueden inflamarse espontáneamente en condiciones ambientales, resultando la combustión espontánea a través de complejos cambios físicos y químicos. En el proceso de almacenamiento se presentan dificultades en el control del aumento de las temperaturas en las pilas de carbón (Asory y Akgun, 2000). Esto es porque el carbón reacciona con el oxígeno del aire y se produce una reacción exotérmica, incluso en condiciones ambientales.

El problema inicia cuando la rata de liberación de calor generado por este proceso es mayor del que se disipa por la transferencia de calor a los alrededores (Arisoy, et al., 2006). Si no se previene con medidas adecuadas, los efectos se manifiestan en escapes térmicos produciendo puntos calientes hasta llegar a la combustión espontánea y posteriormente a los focos incandescentes. Durante los últimos años la región del departamento del Cesar-Colombia, es la mayor productora de carbón a nivel nacional con un volumen superior a 30 millones de toneladas por año. Este carbón mineral se constituye como un producto clave en la balanza comercial y energética del país. Por ende, una zona clave para el desarrollo de investigaciones en esta área (IMC, 2009; UPME, 2008). La Mina el Hatillo ubicada al norte de la cadena montañosa de la Loma con reservas medidas de $57 \mathrm{Mt}$, actualmente produce $2 \mathrm{Mt}$ anuales (UPME, 2005). Uno de los eventos que inciden en la calidad del aire en la zona carbonífera del Cesar es la combustión espontánea del carbón reportada por los monitoreos realizados a esta área (UIS, 2010). Por estas razones el fenómeno ha tenido una importancia fundamental para la empresa. Así como a nivel mundial ha despertado interés para los científicos, varios estudios teóricos y experimentales se han realizado en torno a este fenómeno.

Uno de esos estudios fue el realizado por Zhao et al (2013), en el cual se utilizó el modelo Semenov para evaluar el comportamiento térmico del polvo de carbón y predecir el tiempo de retraso de la autoignición (AIDT) de una reserva de carbón, los resultados demostraron que el método micro-calorímetro puede ser usado como una técnica alternativa prometedora para analizar el comportamiento térmico del carbón y predecir el tiempo de retraso de la autoignición, este es un parámetro importante para evaluar el grado peligroso de los materiales, y es predecido por el modelo de Frank-Kamenetskii. Además, Deng et al (2016) desarrollaron un sistema experimental programable de temperatura autodiseñado para investigar las características de la combustión espontánea de carbón parcialmente oxidado, con los resultados obtenidos se infirió que como el área de superficie incrementa y los microporos constituyen el mayor porcentaje de carbones en carbón oxidado parcialmente se genera un incremento en el área de contacto para la reacción carbón-oxígeno. Por otro lado, Lin et al (2017) utilizó un modelo matemático detallado que combina la ecuación de especies de oxígeno y la ecuación de la energía, con el fin de analizar el potencial de combustión espontánea del carbón, del cual se derivaron un grupo de soluciones analíticas y se obtuvieron estados estacionarios, los cuales podrían ser usados para predecir la tendencia de la combustión espontánea del carbón.

La comprensión del fenómeno es importante para la prevención de puntos calientes en las pilas de carbón (Fierro, et al., 1999). Varios procesos ocurren a bajas temperaturas menores a $100^{\circ} \mathrm{C}$. La reacción entre el carbón y el aire es un proceso complejo de sólido-gas que implica la reacción del oxígeno sobre la superficie porosa del carbón, la oxidación de la pirita, metabolismo microbiano y la absorción-adsorción de vapor de agua (Stracher, 2007). Este trabajo está orientado a identificar las condiciones que conllevan a la combustión espontánea en pilas de carbón en la mina el Hatillo - Cesar, mediante la evaluación analítica del fenómeno, teniendo en cuenta la información meteorológica del sitio de estudio y el análisis fisicoquímico del carbón contenido en las pilas referenciadas para este trabajo. La información obtenida es importante para que los procesos de acopio en la mina, sean reorientados con miras a evitar la combustión espontánea y mantener niveles óptimos en la calidad del mineral.

\section{METODOLOGIA}

Para el modelo de prueba del fenómeno de combustión espontánea, se seleccionó una pila de carbón de la Mina el Hatillo ubicada en el departamento del Cesar - Colombia, en un punto georeferenciado con las 
siguientes coordenadas: 1556860,47 Norte y 1057804,63 Este. La selección de la pila en esta mina es consecuencia de la información sobre la alta frecuencia del fenómeno en los centros de acopio del mineral. Con el fin de obtener información suficiente, se tomaron datos durante 48 horas continuas considerando diferentes aspectos relacionados con las características de la pila y las condiciones atmosféricas. Para la pila se midió la temperatura a unos 25 y $100 \mathrm{~cm}$ de la superficie; para las condiciones circundantes a la pila se recolectó la información meteorológica de la zona de estudio, con el propósito de determinar la incidencia de los factores atmosféricos y los requerimientos del modelo analítico de Frank Kamenestkii, está información corresponde a la radiación solar, precipitación, humedad relativa, temperatura del ambiente y velocidad del aire. Las pilas se midieron con termocuplas Tipo 3825K2RC y Tipo 3850K2RC para diferentes longitudes, instaladas por medio de cables Referencia C0508 y C0811 a un colector de datos Data Logger Testo Tipo TEE 0577 1718, con registro de datos cada 20 segundos, logrando el almacenamiento de la información en 15273 registros por medio de un computador y un software Comfort Testo (Gómez, et al., 2009).

La pila de carbón utilizada para este estudio tiene las siguientes características: Altura de $8 \mathrm{~m}$ con una pendiente de $40^{\circ}$ aproximadamente en las laderas. La cantidad de carbón almacenada es de 10500 Toneladas, ésta pila de carbón presenta mezclas de diferentes mantos con valores caloríficos de 10500 hasta 12000 BTU. Asimismo, se tomaron cuatro muestras de carbón alrededor de 4 kilogramos en varios puntos representativos alrededor de toda la pila y cerca de puntos calientes para la respectiva caracterización. Las propiedades de los carbones del Hatillo pueden ser caracterizados por los análisis próximos y últimos de seis muestras tomadas de la pila objeto del estudio. Estos carbones han sido objeto de estudio y se ha demostrado que tienen buenas propiedades térmicas (Bastidas, et al., 2010), además se han hecho análisis de los carbones de acuerdo con los procedimientos estándar de las Normas: ASTM D-3172, ASTM D-3173, ASTM D-3174, ASTM D-3175, ASTM D 3177 Método B ASTM D 5865 ASTM D 720, ASTM D-3178, ASTM D-3179.

Los fenómenos involucrados que producen la combustión espontánea del carbón en pilas, se observan en la Figura 1. El cambio químico ocurre en la superficie de la partícula y en los poros de la misma generando energía en forma de calor. Luego se presenta el transporte de calor, de masa, vapor de agua y oxígeno. Estos se dan por medio de los mecanismos de transferencia de energía mediante la conducción y convección (Stracher, 2007; Wang, et al., 2003). El proceso en la superficie interna de la partícula consiste en la oxidación a través de los poros, debido a la difusión del oxígeno, provocando efectos importantes en la estructura porosa del carbón, como la oxidación parcial del carbón en la superficie (Wang, et al., 2003) a bajas temperaturas (menores a $100^{\circ} \mathrm{C}$ ) en la que hay formación de $\mathrm{CO}_{2}$ y calor.

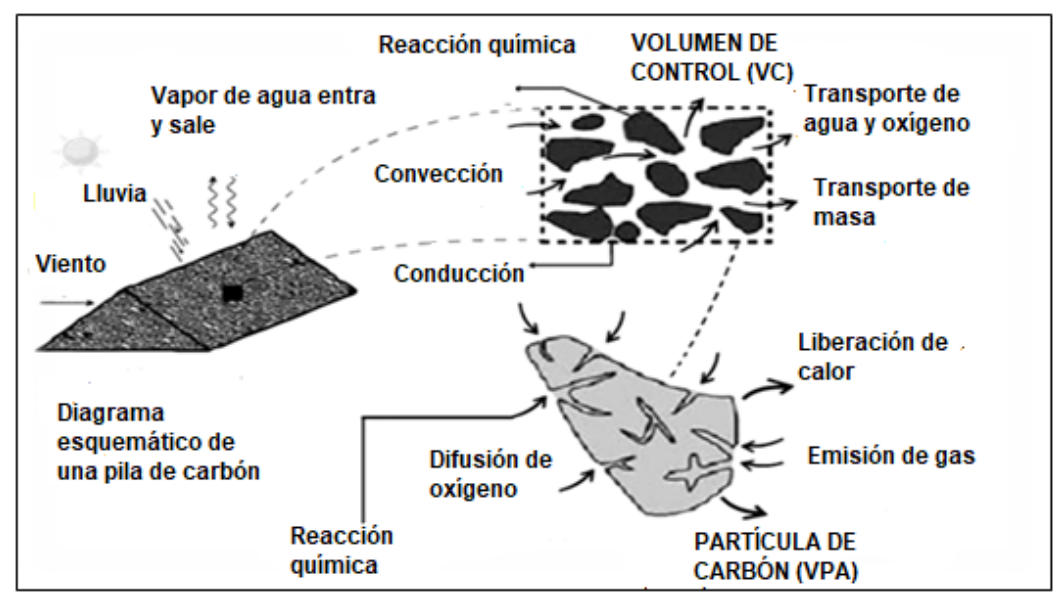

Fig. 1: Sección de una pila de carbón

Esta reacción es complicada y puede consistir de varias etapas y procesos, dependiendo también de la presencia de otras sustancias como el agua y la pirita. La velocidad de oxidación de la reacción depende de varias condiciones. Las más importantes son aportadas por la atmosfera circundantes, teniendo en cuenta para este caso las siguientes: el contenido de oxígeno del aire, temperatura, superficie específica y composición del carbón. Por ejemplo, si tenemos en cuenta un pequeño volumen de carbón y oxígeno, donde la reacción es constante y no está limitado por el suministro de oxígeno, entonces la velocidad de reacción puede ser representada con la ecuación 1, de la forma de Arrhenius:

$$
r=A \cdot F \cdot \exp (-E / R T)
$$

En esta ecuación, $\mathrm{r}$ es la rata de oxidación de la reacción, $\mathrm{F}$ es el contenido de oxígeno del aire, $\mathrm{R}$ es la contante internacional de los gases, T la temperatura y E la energía de activación del carbón. Esta última depende del tipo de carbón y su composición. El factor de frecuencia A, puede ser considerado como una 
medida de reactividad de la matriz del carbón en el proceso de oxidación. Este factor depende de la superficie específica del carbón y tiende a incrementarse si la matriz se vuelve más fina. Los valores de $\mathrm{E}$ y A son obtenidas en laboratorios, sin embargo se tomaron promedios de $54 \mathrm{kJmol}^{-1} \mathrm{y}^{2} \mathrm{~s}^{-1}$ respectivamente, teniendo en cuenta el trabajo de Jones (1996) en el que empleó carbones de características similares en rango a los carbones de la Mina el Hatillo y los cálculos desarrollados por Gómez y otros (2009).

Modelo Matemático de Frank Kamenestkii. La ecuación de la conservación de la energía para los reactivos sólidos infinitos y porosos, excluyendo el consumo del reactivo y la evaporación, se representa de la siguiente forma:

$$
\rho C_{\mathrm{P}} \frac{\partial \mathrm{T}}{\partial \mathrm{t}}=\mathrm{k} \frac{\partial^{2} \mathrm{~T}}{\partial^{2} \mathrm{t}}+\mathrm{Q} \rho \operatorname{Aexp}(-\mathrm{E} / \mathrm{RT})
$$

Donde el término del lado izquierdo de la ecuación es la rata local de cambio de la entalpia en el sólido, el primer término del lado derecho es la transferencia de calor por conducción y el segundo término es la generación de calor de una reacción exotérmica (o varias reacciones que ocurren simultáneamente, pero se considera una reacción global). Frank Kamenetskii en sus estudios relaciona las ecuaciones de generación de calor y los mecanismos de transferencia de energía, estableciendo un parámetro crítico adimensional $\delta_{\mathrm{c}}$ denominado Parámetro de Frank Kamenetskii, utilizado para establecer las condiciones críticas de la combustión espontánea. Este parámetro se define de la siguiente manera (Jones, et. al., 1996):

$$
\delta_{c}=\frac{r^{2} Q \rho A \exp \left(-\frac{E}{\mathrm{RT}_{\mathrm{a}}}\right)}{\mathrm{k}\left(\frac{\mathrm{RT}_{\mathrm{a}}^{2}}{\mathrm{E}}\right)}
$$

Donde $\delta_{c}$ es el parámetro critico que depende de la forma geométrica de los reactivos $\left(\delta_{c}=0.88\right.$ para un bloque, 2.57 para un cubo y 3.531 para un cilindro uniforme). En las ecuaciones 2 y $3 \mathrm{Q}$ es el calor generado, $\mathrm{C}_{\mathrm{P}}$ es el poder calorífico, $\rho$ es la densidad suelta y $T_{a}$ la temperatura ambiente.

El parámetro crítico de Frank Kamentskii es importante en el proceso de almacenamiento porque permite determinar la altura permisible de las pilas en condiciones seguras, sin temor a que se presente el fenómeno de la combustión espontánea. Por ejemplo, para una pila de carbón en forma cubica el valor crítico de $\delta_{c}=$ 2.57, este parámetro crítico indica que por encima de esta cantidad existe peligro de ignición, por el contrario si la cantidad es menor no hay cabida a la combustión.

Anteriormente, se presenta una alternativa para evaluar la altura ideal de las pilas de carbón sin presencia de la combustión espontánea. Sin embargo es importante conocer el tiempo de ignición de estos carbones. Esta aplicación es muy utilizada como criterio de seguridad para el transporte del carbón en buques, silos y en los vagones de los trenes. Los cálculos del tiempo de ignición están llenos de dificultad porque dependen en gran medida del grado de súpercriticidad; es decir, las zonas críticas de combustión (Jones, 2000). Se obtuvo una expresión para el tiempo de ignición de un reactivo (carbón) con base en parámetros dependientes e independientes a la pila de carbón, tales como: energía de activación, factor de reactividad, densidad para cualquier temperatura, temperatura ambiente, constante de los gases. Esto se puede aplicar en los centros de almacenamientos de carbones por sus propiedades cinéticas, físicas y químicas. La ecuación se expresa de la siguiente forma:

$$
\mathrm{t}_{\mathrm{IG}}=\frac{\mathrm{RT}_{\mathrm{a}}^{2}}{\mathrm{E}} \frac{\mathrm{c}}{\mathrm{QA}} \exp \left(-\mathrm{E} / \mathrm{RT}_{\mathrm{a}}\right)
$$

Donde $c$ es la capacidad calorífica y las demás variables son iguales a las citadas en la sección anterior, considerando los resultados de las variables cinéticas, térmicas, físicas y químicas del carbón descritas anteriormente, se presenta una simulación del parámetro critico de Frank kamenetskii para determinar la altura optima de las pilas de carbón sin efectos de la combustión espontánea evaluada a diferentes niveles de temperatura ambiente, de igual forma, se involucra el tiempo de ignición de estos carbones.

\section{RESULTADOS}

Inicialmente se relacionan en las tablas 1 y 2, los resultados de la caracterización de las muestras de carbón tomadas de las pilas. Entre los indicadores de calidad del carbón, el poder calorífico oscila entre 6038 y 6850 $\mathrm{cal} / \mathrm{g}$, las cenizas entre 1.8 y $10.4 \%$ y la humedad entre 8.8 y $11.2 \%$, lo que indica un carbón de buena calidad térmica, propenso a alcanzar los límites críticos para la combustión espontánea. Sin embargo, estos parámetros no son suficientes para inferir sobre la sensibilidad de las variables que generan en las pilas de 
carbón este fenómeno, agrupados por Kamenetskii en el tiempo de acopio del mineral y el tamaño de la pila. En este sentido, se hace una valoración de estos variables para establecer sobre cual se debe intervenir para disminuir el riesgo a la combustión espontánea.

Tabla 1: Análisis próximos (Base seca, 1mm: libre de materia mineral)

\begin{tabular}{cccccc}
\hline Muestras & (\%) Humedad & (\%)Ceniza & $(\%) M V$ & P. Cal(cal/g) & $(\%) S$ \\
\hline A & 8.8 & 2.0 & 38.0 & 6850 & 0.73 \\
B & 9.4 & 3.5 & 37.5 & 6618 & 0.70 \\
C & 9.4 & 2.5 & 36.8 & 6742 & 0.69 \\
D & 9.9 & 5.7 & 36.1 & 6431 & 0.45 \\
E & 9.8 & 10.4 & 34.8 & 6038 & 0.59 \\
F & 11.2 & 1.8 & 37.9 & 6588 & 0.45 \\
\hline
\end{tabular}

Tabla 2: Análisis elementales

\begin{tabular}{ccccc}
\hline Muestras & $(\%) C$ & $(\%) H$ & $(\%) N$ & $(\%) O$ \\
\hline A & 69.39 & 5.65 & 1.23 & 12.74 \\
B & 67.18 & 5.54 & 1.29 & 15.00 \\
C & 68.47 & 5.59 & 1.27 & 13.68 \\
D & 65.35 & 5.56 & 1.19 & 16.91 \\
E & 61.43 & 5.26 & 1.14 & 21.18 \\
F & 66.67 & 5.73 & 1.34 & 15.27 \\
\hline
\end{tabular}

La densidad suelta de los carbones de la Mina el Hatillo varía entre 800 y $950 \mathrm{~kg} \mathrm{~m}^{-3}$. Las propiedades térmicas como la capacidad calorífica se encuentra alrededor de $1260 \mathrm{~J} \mathrm{Kg}^{-1} \mathrm{~K}^{-1}$, Los valores de la conductividad térmica es de $0.1998 \mathrm{~W} \mathrm{~m}^{-1} \mathrm{~K}^{-1}$. Estos valores son tomados de otros estudios a nivel internacional (Yuan y Smith, 2009), como referencia ya que las características de estos carbones son similares en rango y se pueden comparar permitiendo una evaluación rápida de las propiedades influyentes en el fenómeno de la combustión espontánea del carbón. En la Figura 2, se observa el comportamiento de la temperatura en una pila de carbón a dos profundidades registrados por el colector de datos y confrontadas con las variables atmosféricas.

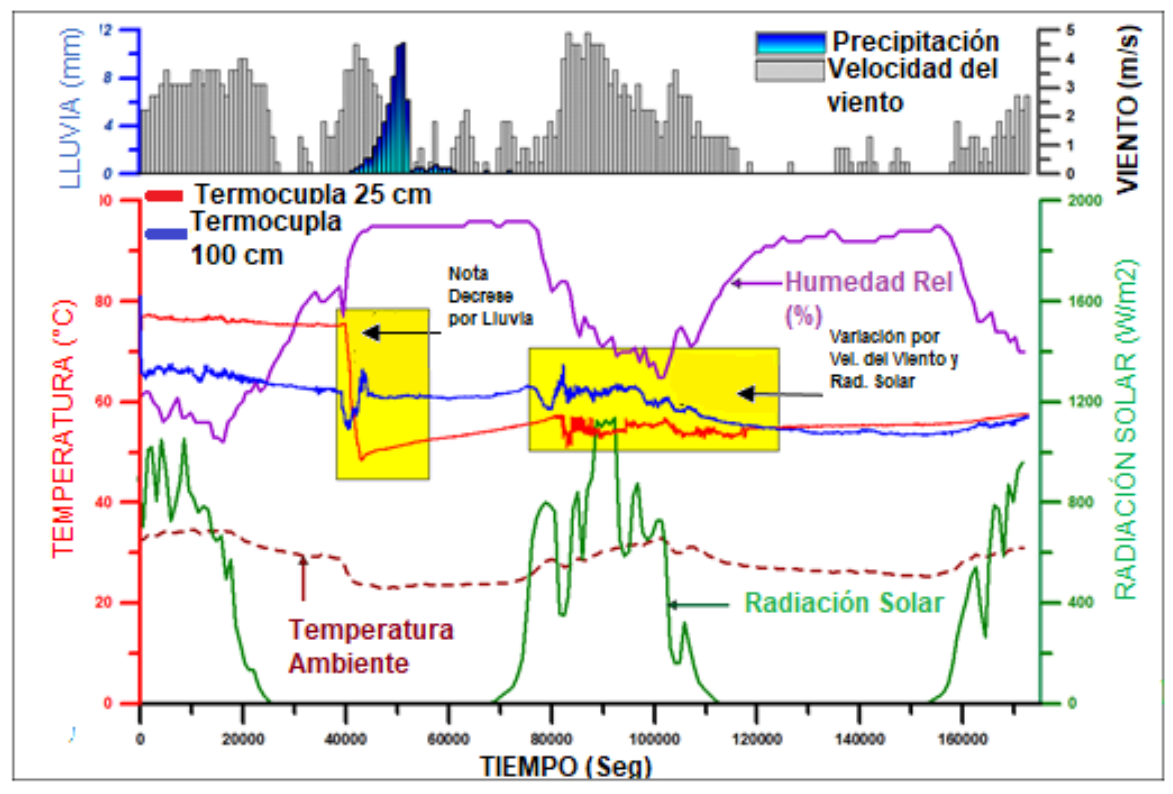

Fig. 2: Perfil térmico de una pila de carbón Vs variables meteorológicas

Como se observa en la figura 2, inicialmente los perfiles térmicos en ambas termocuplas, muestran comportamientos diferentes, esto es adjudicado a que la pila de carbón tiene mayores niveles de temperatura en la parte superficial que en profundidad, puesto que es el área que tiene mayor exposición a factores atmosféricos. Luego, se puede apreciar la disminución de la temperatura debido al comienzo del periodo nocturno y precipitación (18:00 pm), lo que implica un aumento de la humedad relativa, ausencia de la 
radiación solar y disminución de la temperatura ambiente; es decir, en este periodo de tiempo es apenas lógico que se reduzca la temperatura de la pila de carbón. Cerca del mediodía (12:00 pm) se incrementa la temperatura debido al aumento de la radiación solar, la velocidad del aire y la temperatura ambiente. Por el contrario disminuye la humedad relativa. Durante esta etapa hay una disputa por el oxígeno del aire en el exterior de la pila y en diferentes profundidades. Por último, es importante resaltar que la temperatura de las pilas de carbón es superior a la temperatura local del ambiente.

En la figura 3, se reportan los resultados de la simulación de la altura permisible de las pilas de carbón como parámetro de seguridad y prevención de la combustión espontánea, asimismo el tiempo de ignición de estos carbones también es relacionado mediante el mismo procedimiento de simulación. Estos parámetros comparten el mismo intervalo de temperatura para el modelamiento a diferentes temperaturas dentro del rango de $300^{\circ} \mathrm{K}$ a $440 \stackrel{\circ}{\mathrm{K}}$. La simulación ofrece resultados del comportamiento de estas variables cuando son sometidas a diferentes temperaturas.

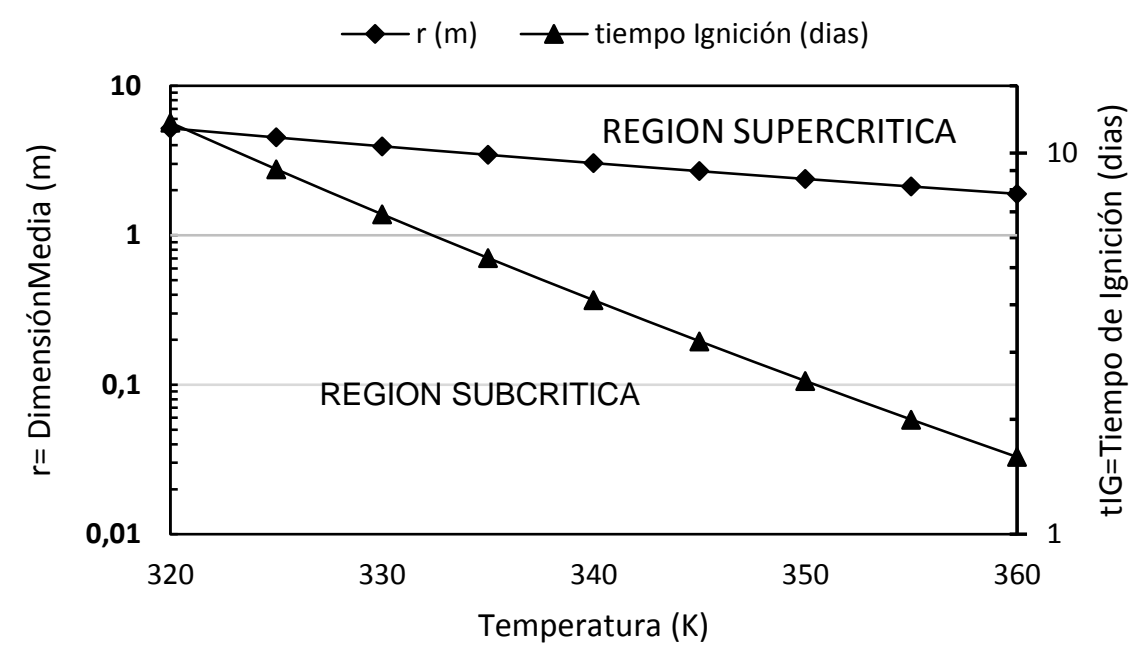

Fig. 3: Simulación del Tamaño óptimo de las Pilas de Carbón y el Tiempo de Ignición.

Tres variables se relacionan entre sí, las cuales son: Dimensión media en (metros) y el tiempo de encendido (días) de los carbones acopiados de la Mina El Hatillo, ambas variables dependen de las temperaturas a las cuales son expuestos. La dimensión media es obtenida a partir del parámetro de seguridad de Frank Kamenetskii y su principio de ignición térmica; de igual manera, el tiempo de ignición de los carbones por medio de la literatura (Jones, 2000). En la gráfica se presentan dos regiones denominadas supercriticas y subcriticas delimitadas por las curvas de ambas variables las cuales simplemente manifiestan las zonas de riesgo de ignición y zonas seguras donde no ocurre ignición; es decir, para las dimensiones medias de los carbones acopiados si se encuentran por encima de la curva sindica que estos carbones están en riesgo de presentar la auto-combustión, de igual forma, para el tiempo de ignición. Cuando se almacenan los carbones por un tiempo mayor al limitados en la curva de tendencia estos son propensos a la ignición espontánea, para en entender un poco estas aplicaciones se presenta a continuación un ejemplo en los procesos de almacenamiento del carbón. Este estudio se refiere a los carbones de la mina el Hatillo, donde es de gran importancia utilizar los resultados obtenidos para las operaciones de almacenamiento teniendo presente la altura optima de las pilas y el tiempo de ignición de los carbones acopiados como parámetros de seguridad.

A continuación se presenta una alternativa para las metodologías de almacenamiento y despacho de carbón en los centros de acopio. Por ejemplo, los carbones que van demorar mucho tiempo almacenados superior a 2 días, lo cual es el límite del tiempo de ignición para temperaturas ambientales. Esto señala que si superar este intervalo de tiempo va a presentar focos incandescentes provenientes de la combustión espontánea. Por tanto, deben ser almacenados bajo las condiciones seguras; es decir el tamaño de las pilas no deben ser superiores a $1 \mathrm{~m}$. En la figura 4 se puede observar la variación del tamaño de los carbones acopiados y el tiempo de ignición a diferentes temperaturas. Por el contrario, cuando se conoce que tiempo de los carbones almacenados no va hacer superior al límite de ignición, se pueden acopiar los carbones con alturas mayores a $1 \mathrm{mts}$, en este caso el tamaño de las pilas no es relevante si el tiempo de los carbones acopiados demora lo justo. De esta forma se involucran los criterios del tiempo de ignición y el tamaño óptimo de las pilas de carbón durante el acopio en las metodologías de almacenamiento para prevenir el fenómeno de la Combustión espontánea y hacer relaciones con estos parámetros en los procesos de acumulación y despacho. 


\section{DISCUSIÓN}

Esta investigación contribuye a la literatura y al conocimiento científico, debido a que brinda un parámetro óptimo sobre el cual se puede disminuir el riesgo de ignición de los carbones, en función del tiempo de almacenamiento y la altura de las pilas. Los resultados evidenciaron que la combustión espontánea del carbón durante el acopio es causado por variables intrínsecas (Naturaleza del Carbón) como: Rango del Carbón, Contenido de Pirita, Humedad Inherente, Tamaño y superficie de la partícula, constituyentes petrográficos, químicos y físicos; esto es muy similar a lo obtenido por Casas, Blandón y Molina (2014), quienes analizaron los factores más importantes y con influencia directa sobre el polvo de carbón, los análisis mostraron que la granulometría es un factor muy importante como indicador de la explosividad del polvo de carbón, debido a que cuando las partículas son más finas, el material es más susceptible y se aumenta el riesgo de generar una explosión tras otra, con un área mayor de afectación; además, la madurez del carbón se constituye en otro factor importante a considerar en la prevención de la auto-combustión y la explosividad, ya que un carbón joven presenta elementos muy susceptibles e inestables a las altas temperaturas, al combinarse esto con el contenido de materia volátil y algunos otros ya influyentes en el ambiente, tales como: las corrientes de aire, la concentración del polvo, la humedad y la presión, entre otros, crean un entorno ideal para dar lugar a la combustión y luego a una explosión violenta que afectará una gran porción del área donde se produzca.

En este estudio se observó que este fenómeno también es afectado por variables extrínsecas (atmosfera y condiciones mineras) como: temperatura, humedad relativa, concentración de oxígeno, radiación solar, flujo aire, precipitación y condiciones de acopio; esto afirma lo planteado por Taborda-Acevedo, Jurado y Cortés (2016) quienes evaluaron el efecto del glicerol en la inhibición de auto-combustión del carbón como reductor de re-adsorción de agua, mediante la adición de glicerol en diferentes cantidades, los resultados mostraron que la adición de glicerol a carbones disminuye la humedad, lo que resulta en la disminución de la readsorción de agua en comparación con el carbón seco, estos dos hechos implican un aumento en las propiedades del carbón en los procesos térmicos, los resultados también implican que la auto-combustión de carbón se inhibe, esto fue demostrado mediante el cálculo de la velocidad de combustión espontánea, obteniendo el valor más bajo de la reactividad de las muestras analizadas, lo que evidencia que existe una relación directa entre la capacidad de adsorción de agua y el riesgo potencial de la combustión espontánea

\section{CONCLUSIONES}

De los resultados obtenidos en este estudio se pueden destacar las siguientes conclusiones: 1) la fenomenología de la combustión espontánea se le atribuye a una reacción química entre el carbón y el oxígeno, generando energía en forma de calor, que es transportada por los mecanismos de transferencia de energía en una matriz porosa de carbón. 2) las condiciones de almacenamiento de los carbones de la mina el hatillo no deben superar $1 \mathrm{mt}$ de altura con el fin de minimizar el fenómeno de la combustión espontánea, estos resultados son obtenidos de la simulación del parámetro de Frank Kamenetskii y propiedades físicas y químicas de los carbones. 3) el tiempo de ignición de los carbones de la mina el Hatillo es de 2 días a la intemperie, estos resultados son obtenidos del modelamiento del tiempo de ignición de J.C. Jones y las propiedades físicas y químicas de los carbones.

\section{NOTACION}

$\begin{aligned} Q & =\text { Poder Calorífico } \\ P & =\text { Densidad Suelta } \\ A & =\text { Factor Pre-Exponencial } \\ E & =\text { Energía de Activación } \\ R & =\text { Constante de los Gases } \\ \delta & =\text { Parámetro de seguridad } \mathrm{F}-\mathrm{K} \\ \Lambda & =\text { Conductividad Térmica } \\ r & =\text { Longitud media en condiciones } \\ T_{a} & =\text { Teguras } \\ c & =\text { Capacidad Calorífica } \\ t_{I G} & =\text { Tiempo de Ignición }\end{aligned}$

\section{AGRADECIMIENTOS}

Los autores agradecen al Ingeniero de Minas Joe Michael Gómez por el significativo aporte y apoyo a este trabajo. 


\section{REFERENCIAS}

Arregocés, H., R. Rojano, L. Angulo y G. Restrepo, Predicción y análisis de la contribución de PM10 desde pilas de carbon en una mina a cielo abierto, doi: 10.4067/S0718-07642016000400010, Inf. Tecnol., 27 (4), 93-102 (2016)

Asory, A. y F. Akgun, Effect of pile height on spontaneous heating of coal stockpiles, Combustion Science and Technology, 157-168 (2000)

Arisoy, A. y F. Akgun, Modelling of spontaneous combustion of coal with moisture content included, Fuel, 73, 281-286 (1994)

Arisoy, A., B. Beamish y E. Cetegen, Modelling Spontaneous Combustion of Coal, Turkish J. Eng. Env. Sci, 30, 193-201 (2006)

Bastidas, M., R. Bermudez, P. Jaramillo y F. Chejne, Optimización Termoeconómica y Ambiental usando Algoritmos Genéticos Multiobjetivo, 21(4), 35-44 (2010)

Carras, J. y B. Young, Self-Heating of coal and related materials: Models, application and test methods, Energy Combustion, 20, 1-15 (1994)

Casas, E., A. Blandón y J. Molina, Evaluación de parámetros para determinar el grado de explosividad del polvo de carbón, Boletín de Ciencias de la Tierra, 36, 42-54 (2014)

Deng, J., J. Zhao, Y. Zhang, H. Anchi, X. Liu, X. Zhai y C. Wang, Thermal analysis of spontaneous combustion behavior of partially oxidized coal, Process Safety and Environmental Protection, 104, 218-224 (2016)

Fierro, V. y otros autores, Prevention of spontaneous combustion in coal Stockpiles Experimental results in coal storage yard, Processing Technology, 59, 23-34 (1999)

Gómez, J., y otros autores, Determinación de perfiles de temperatura de una pila de carbón enmarcada en la etapa III llamada "trabajo de campo a nivel experimental" del proyecto de investigación "Estudio de la combustión espontánea de los carbones del yacimiento el Hatillo - Cesar, CIDTEC, 2-64 (2009)

IMC, Información Minera Colombiana, Producción Colombiana de carbón por departamentos, Bogotá, Colombia (2009)

Jones, J., On the role of times to ignition in the thermal safety of transportation of bituminous coals, Fuel, 1561-1562 (2000)

Jones, J., y otros autores, Kinetic parameters of oxidation of bituminous coals from heat-release rate measurements, Fuel, 1755-1757 (1996)

Lin, Q, S. Wang, Y. Liang, S. Song y T. Ren, Analytical prediction of coal spontaneous combustion tendency: Velocity range with high possibility of self-ignition, Fuel Processing Technology, 159, 38-47 (2017)

Pone, J. y otros autores, The spontaneous combustion of coal and its by-products in the Witbank and Sasolburg coalfields of South Africa, Coal Geology, 1-17 (2007)

Stracher, G., Geology of Coal Fires: Case studies from Around the World. Usa: The Geological Society of America, 53-70 (2007)

Taborda-Acevedo, E., W. Jurado y F. Cortés, Efecto del glicerol en la inhibición de la combustión espontánea de carbón sub-bituminoso, Boletín de Ciencias de la Tierra, 40, 64-74 (2016)

UIS, Universidad Industrial del Santander, Informe de operación del sistema especial de vigilancia de calidad de aire en la zona carbonífera del departamento del Cesar, 2-92 (2010)

UPME, Unidad de Planeación Minero Energética, Boletín estadístico de minas y energía, Ministerio de Minas y Energía, 25-45, Bogotá, Colombia (2008)

UPME, Unidad de Planeación Minero Energética. La cadena del Carbón, El carbón Colombiano: Fuente de Energía para el Mundo, Ministerio de Minas y Energía, 7-52, Bogotá, Colombia (2005)

Wang, H., y otros autores, Coal oxidation at low temperatures: oxygen consumption, oxidation products, reaction mechanism and kinetic modeling, Progress in Energy and Combustion Science, 29, 487-513 (2003)

Yuan L. y A. SMITH, CFD, Modeling of spontaneous heating in a large-scale coal chamber, Journal of Loss Prevention in the Process Industries, 426-433 (2009)

Zhao, X, Q. Wang, H. Xiao, Z. Mao, P. Chen y J. Sun, Prediction of coal stockpile autoignition delay time using microcalorimeter technique, Fuel Processing Technology 110, 86-93 (2013) 